\title{
ELECTRON TRANSPORT IN METALS AND SOLIDS CONFERENCE IN OTTAWA
}

A $N$ international conference on "Electron Transport in Metals and Solids" was held in Ottawa during September 10-14. The meeting was arranged by Dr. D K. C. MacDonald, of the National Research Council of Canadza, and was supported by the International Union of Pure and Applied Physics, by Unesco, and by the National Research Council. The décor of the meeting-the National Research Council Laboratories, overlooking the wide Ottawa Riverwas much appreciated by the fifty or so participants. By skilfully rebutting all attempts to include too many persons and too many papers, Dr. MacDonald and his assistants kept the programme within bounds, allowing time for proper consideration of the main topics and discussion of the problems still unsolved. One could wish that all scientific conferences were organized so sensibly, and not made indigestible with a gobble of successive pemmican pellets of condensed yet incoherent discourse.

A full account of the proceedings is to be published as a special number of the Canadian Journal of Physics. 'The following notes summarize (in logical, but not chronological, order) the main subjects.

J. Bardeen opened with a survey of the state of knowledge on interactions between electrons and lattice vibrations, leading to a lively discussion of the causes and effects in the lattice-scattering part of the resistivity of metals. It seems that, with corrections to the Bloch theory, this problem is now understood in principle, but detailed calculations of the resistivity of even simple metals are only now beginning to look reliable. The effects of deviations from equilibrium in the phonon distribution were discussed by E. H. Sondheimer, with reference to recent observations on resistivity and thermoelectric phenomena at low temperatures. G. Borelius pointed out that the resistivity of pure metals at high temperatures can be correlated phenomenologically with other properties ; it appears that there may be much to learn from measurements of conductivity of liquid metals. The relation between X-ray scattering and electron scattering by lattice waves was developed by Sir K. S. Krishnan in an ingenious argument.

A review of electrical trarsport phenomena in alloys, by J. Friedel, brought out the wide variation of behaviour found with various transition elements, both in large and small concentrations, but most of the facts could be correlated reasonably well with theory. More controversial was the discussion concerning the minimum (and sometimes, also, the maximum) in resistance found at low temperatures in metals containing certain impurities. Papers by C. J. Gorter, by R. W. Schnitt and by J. Korringa showed the complexity of the phenomenon and the perplexity of its interpreters. It seems that there may be two distinct mechanisms, one related to an antiferromagnetic ordering in the spins of $d$-electrons in impurities from the transition group of elements and the other, not yet understood, which seems to require some sort of energy-level very near the Fermi surface of the matrix metal.

P. $\mathrm{x}$. Klemens gave an account of the calculation of the electrical resistance due to lattice imperfections in metals. Although some reasonable estimates can be made for the scattering by dislocations, there are still difficulties in explaining the large changes in resistance caused by cold work in metals. An interpretation in terms of scattering by bands of stacking faults was supported by A. Seeger; but in the discussion other speakers found difficulty in believing that this effect could be large.

More fundamental problems concerning the validity of the Boltzmann equation, and the corrections one could make to it, were discussed by I. Prigogine, who outlined recent work on the statistical mechanics of irreversible processes. It is hoped that by starting from the 'master equation', more refined calculations may shortly be possible. Within the framework of the Boltzmann equation, it was shown by J. M. Ziman that there is a close connexion between the thermodynamics of irreversible processes and the variational principle of Kohler, which may be exploited to simplify calculations of transport coefficients. Another variational principle for conduction phenomena in the presence of a magnetic field or asymmetric scattering mechanism was proposed by A. Seeger, and R. Kubo gave a general expression for the conductivity tensor, derived directly from dispersion theory applied to the density matrix.

In reporting measurements on the electrical and thermal conductivity of metals, K. Mendelssohn emphasized the interest of thermal conduction in superconductors, which is effectively pure lattice conduction (although C. J. Gorter pointed out that the two-fluid model could be interpreted in such a way as to lead to an appreciable contribution from a circulation of normal and superfluid electrons). Information from lattice conduction could, as pointed out by $P$. G. Klemens, supplement electrical measurements in identifying the various types of imperfections in metals. Other experimental data were given by G. K. White, reporting on work in progress at the National Research Council Iaboratories.

Nuclear magnetic resonance and the electronic structure of conductors was the subject of a review by $\mathrm{N}$. Bloembergen. It is evident that measurement of the Knight shift yields very diverse information, some of which may be related to band structure or to known transport properties. There seems room for better correlation between this study and measurements of the more traditional transport parameters. On the theoretical side, D. Pines brought up to date the theory of plasmons, showing that in most solids the valence electrons form a plasma the collective oscillations of which have important effects. The calculations are in remarkably good agreement with experiment. It was suggested that, where there are present both holes and electrons, as in a semiconductor, there may be new forms of excitation, having dispersion properties like those of a sound wave; these 'distinct electron modes' may be literally named 'demons'. The various techniques by which direct information can be obtained about the geometry of the Fermi surface were described by R. G. Chambers. Cyclotron resonance, the anomalous skin effect, and the de Haas - van Alphen effect can 
all show up anisotropy, and attempts are being made to interpret the results quantitatively.

Although largely devoted to the study of metals, the conference spent some time on topics related to semiconductors. N. F. Mott presented his theory of the transition to metallic conduction in semiconductors, regarding electronic transport in an impurity band as the jumping from site to site with activation by lattice vibrations. P. R. R. Aigrain, on the other hand, preferred a collective picture, in which the randomness of the distribution of impurity sites plays an important part. He suggested that certain states, although not localized, cannot carry current, and hence explained the low mobility in the impurity band. Experimental date on galvanomagnetic effects in $n$-type InSb at $4^{\circ} \mathrm{K}$. were given by $\mathrm{H}$. P. R. Frederikse. By considering valence bonds in various compounds, E. Mooser and H. W. B. Pearson were able to present some rules which allow the prediction of semiconducting properties.

An important contribution was made by A. F. Joffé, who discussed recent Russian work on heat conduction in semiconductors. Besides lattice conduction (which may be reduced very effectively by using mixed crystals so that phonons are scattered by the random distribution of atomic mass), there is a significant electronic contribution, and also a further part which may be due to transport of heat by excitons. This led to considerable discussion, with opinions divided as to the exact interpretation. J. M. ZIMAN

\section{GAS TURBINE DEVELOPMENT}

$\mathrm{T}$

HE Engineering Department of the North Herts Technical College, Letchworth, arranged a conference on "Gas Turbine Development", which was held during October 20-21. It attracted more than a hundred, most of whom were leading designers or research workers in the appropriate field.

The first of the four main papers was given by Mr. G. B. R. Feilden, engineering director, Messrs. Ruston and Hornsby, Ltd., who offered a brief résumé of the development of the Ruston and Hornsby gas turbine $(750 / 1,000 \mathrm{~kW}$.$) , since 1946, dealing parti-$ cularly with the engineering difficulties which were experienced in putting the research designs into working order. At the end of $1,500 \mathrm{hr}$. running experience with this machine, it was decided to go ahead with a production model capable of $750 \mathrm{~kW}$. and rising to $1,000 \mathrm{~kW}$. maximum output. Improvements were made in the prototype output by raising the maximum temporature, and increasing the length of the turbine blades by 7-8 per cent; production economy being maintained by utilizing tooling for existing blades. It was decided, also, to improve the matching of the components and make minor adjustments to the burners. The completed model had a compressor and turbine practically identical with the prototype, though epicyclic gears had been introduced and the turbine mounting improved. In order to enable the individual components to be serviced easily, however, considerable modifications in layout had been incorporated.

Mr. Feilden illustrated developments in the more recent installations, including one at Milan, which has now been in continuous operation for $10,000 \mathrm{hr}$. This machine is fired by natural gas, and the arrange- ment of the combustion chambers with ancillary ducting has proved very practicable for servicing. He also illustrated the installation at Debden, in which the heat exchanger is placed below the set, and the combustion chamber is arranged at an angle downwards. This is a coal-tar fuel-burning set with the ducting arranged upwards. Another slide showed the installation at the Admiralty Test House at Pyecroft, which was made to exacting specifications, the air inlet in this installation being at an unusual angle, and the ducting was woven through the installation. The turbine is to be fitted with a heat exchanger made of corrugated steel plates in the near future.

Mr. Feilden concluded his address by comparing the overall efficiencies of various plants required to produce both power and process steam. The Diesel is the most efficient power producer, and, for a $1,000-\mathrm{kW}$. electrical output, used in conjunction with an exhaust heat boiler, it gives better figures than any other plant for outputs of process-steam up to $7,500 \mathrm{lb}$. per hr.; above that requirement, the gas turbine is better. An exclusively steam plant, even with a highly efficient steam turbine and a high. pressure water-tube boiler, is far behind in overall efficiency, and only begins to be competitive if a very considerable quantity of steam (more than 20,000 lb. per hr.) is asked for.

Discussion revealed that problems of servicing under varying climatic and atmospheric conditions have been extremoly interesting. While the installation at Kuwait has to digest large quantities of sand, this appears to have no adverse effect upon the turbine blades. The sodium sulphate in airborne particles presents an entirely different problem with the Milan installation, and it is necessary to wash the blades every 200-300 hr. with a solution of 'Teepol' and water; the turbine has now done $3,000 \mathrm{hr}$. non-stop.

"The Marine Gas Turbine" was the subject of the second paper, by A. W. Pope, chief gas turbine engineer, Messrs. W. Allen, Sons and Co., Ltd. Mr. Pope showed that there are two distinct applications of the marine gas turbine-naval and merchant marine. In connexion with naval applications, there are many differing requirements. Naval can be divided into two categories: (a) protection of convoys, and (b) task forces. The former duty is performed mainly by frigates, a comparatively new class of ship of which there are various types (fast escort, anti-submarine, anti-aircraft and air direction). The task force duties are undertaken by aircraft carriers and cruisers. The varying requirements mean that aircraft carriers spend much of their time at full power, whereas frigates spend very little. On one such ship, it was found that 70 per cent of full power was used for only 1 per cent of the ship's life. 'There is here an obvious application for gas turbines (known as boost turbines) to provide the extra power when required. Already two British ships have been provided with such turbines, and an American ship is powered by 9,000 steam h.p., and 21,000 gas turbine h.p., with a saving of 20 per cent on engine weight.

Gas turbines are also being used for electrical generation. There are two types of generator set, namely, emergency and base-load. In emergency sets, fuel consumption is unimportant, and the gas turbine correspondingly simple. Mr. Pope gave an example of the development of such a turbine, detailing various changes in design to give greater efficiency. 